\title{
Influence of Laser Modulation Frequency on the Performance of Terahertz Photoconductive Switches on Semi-Insulating GaAs Exhibiting Negative Differential Conductance
}

\author{
Gaudencio Paz-Martínez ${ }^{\circledR}$, Carlos Gerardo Treviño-Palacios ${ }^{\circledR}$, Joel Molina-Reyes ${ }^{\circledR}$, Senior Member, IEEE, \\ Alejandra Romero-Morán ${ }^{\circledR}$, Eric Cervantes-García, Javier Mateos ${ }^{\circledR}$, Member, IEEE, \\ and Tomás González ${ }^{\circledR}$, Senior Member, IEEE
}

\begin{abstract}
In typical terahertz time-domain spectroscopy systems, the use of the lock-in technique is necessary because of the low current induced at the receiver so that the laser pump beam must be modulated (chopped) at a frequency much lower than the laser repetition rate. This work shows that, in the case of semi-insulating GaAs (SI-GaAs) antennas, this modulation has an important effect on the antenna current and consequently, on the radiated electromagnetic pulse. There exists a threshold bias (whose value depends on the chopping frequency) where an abrupt increase in the current and consequently, in the terahertz emission takes place. The calculated energy of the pulse below and above the threshold shows that the energy doubles. The exact bias voltage at which this occurs changes with the laser modulation frequency when this is below $350 \mathrm{~Hz}$, but at higher frequencies, the threshold remains almost constant. The experiments show that the responsibility for this behavior is the $\mathrm{S}$-shape negative differential conductance exhibited by SI-GaAs originated by a slow field-enhanced charge trapping mechanism, which is also an important source of noise at the receiver of the system.
\end{abstract}

Index Terms-Negative differential conductance (NDC), photoconductive switch, terahertz time-domain spectroscopy (THz-TDS).

\section{INTRODUCTION}

$\mathbf{O}$ VER the past years, there has been an increase of new applications at terahertz $(\mathrm{THz})$ frequencies for which

Manuscript received January 22, 2021; revised May 11, 2021; accepted May 11,2021. Date of publication May 26, 2021; date of current version September 2, 2021. This work was supported in part by Spanish MINECO and FEDER under Project TEC2017-83910-R and in part by Junta de Castilla y León and FEDER under Project SA254P18. The work of Gaudencio Paz-Martínez was supported by Junta de Castilla y León and FEDER for the granted Postdoctoral Fellowship under Project SA254P18. (Corresponding author: Gaudencio Paz-Martínez.)

Gaudencio Paz-Martínez, Javier Mateos, and Tomás González are with the Department of Applied Physics, University of Salamanca, 37008 Salamanca, Spain (e-mail: gaupaz@usal.es; javierm@usal.es; tomasg@usal.es).

Carlos Gerardo Treviño-Palacios, Joel Molina-Reyes, Alejandra RomeroMorán, and Eric Cervantes-García are with the National Institute of Astrophysics, Optics and Electronics, Puebla 72840, México (e-mail: carlost@inaoep.mx; jmolina@inaoep.mx; alejandraromeromoran@gmail.com; eric.cervantes@inaoep.mx).

Color versions of one or more figures in this article are available at https: //doi.org/10.1109/TTHZ.2021.3083926.

Digital Object Identifier 10.1109/TTHZ.2021.3083926 pulsed-laser excited photoconductive switches based on the ultrafast semiconductors are commonly used as broadband $\mathrm{THz}$ power sources. The most widely used semiconductors are low-temperature grown GaAs (LT-GaAs), semi-insulating GaAs (SI-GaAs), and InGaAs because of their properties, such as ultrashort recombination times and high breakdown fields [1]. In order to reduce the cost of such THz systems, SI-GaAs is a promising option because it is cheaper than LT-GaAs and InGaAs, with the disadvantages of higher dark current and longer recombination times. Over the past times, technological efforts to improve the properties of this semiconductor have been explored, such as ion implantation [2], [3] or surface ablation using femtosecond laser [4].

The physics behind the $I-V$ characteristics of SI-GaAs and other high-resistivity semiconductors are well known since long ago [5]-[7]. They show four principal regions, each one presenting a special carrier-transport regime. The low-voltage region is linear (ohmic response). In the second one, the current has a $V^{2}$ dependence associated with space-charge-dominated transport. For higher applied bias, one enters the third region, where negative differential conductance (NDC) is present and current oscillations may take place [8]. Above that voltage range, breakdown is reached, producing a sudden increase of the current phenomenon associated with impact ionization [9], [10].

The semi-insulating characteristic of SI-GaAs is caused by EL2 midgap traps ( $0.75 \mathrm{eV}$ below the conduction band), which compensate shallow donors and acceptors. EL2 is neutral when it is filled and singly or doubly charged when it is empty [11], [12]. It is well known from the literature that the field-enhanced capture of electrons by native EL2 centers is responsible for NDC in trap-dominated SI-GaAs [11]-[13]. The NDC region can have S-shape (SNDC), N-shape, or a combination of both [14]. In the case of SNDC, the voltage-controlled measurement of the $I-V$ curve cannot reveal the S-shape, and only a sharp current increase is observed [15], [16]. NDC in SI-GaAs samples can lead to the appearance of current oscillations associated with propagating the high-field domains, whose frequency depends on the applied field, similarly to what happens in Gunn diodes. However, Gunn effect plays no role on the formation of 
domains in SI-GaAs. Indeed, Gunn oscillations are originated in n-type doped GaAs by the transfer of electrons from the lower high-mobility valley to the upper lower mobility ones, which is a completely different and much faster process than the field-enhanced capture of electrons by deep traps responsible for the NDC in SI-GaAs [12], [19]. Indeed, while Gunn oscillations take place at microwave frequencies, NDC-related current oscillations in SI-GaAs have much lower frequencies [low-frequency oscillations (LFOs)] [17], [18]. The observed LFOs have frequencies between subhertz and kilohertz.

In the work of Rajbenbach et al. [19], the experimental results show that under ac bias, the high-field domains in SI-GaAs are stationary and periodically distributed, the period being electrically controlled by the frequency of the ac voltage. The chopping of the light illumination has also a considerable effect on the LFOs in SI-GaAs because the domains can be controlled and generated artificially through the temporal variations of the optical beam [20]. The NDC region of the $I-V$ curve can also lead to other complex nonlinear phenomena; for example, in experiments with Ge and GaAs excited with modulated far-infrared radiation, the samples can present the chaos and generation of broadband noise [21]. Therefore, a trap-dominated semiconductor between two metal contacts may become a nonlinear system [11] with an external control parameter in the form of high values of the bias voltage, the frequency of the ac bias, or the light modulation frequency, as illustrated in the previous examples.

The trapping and detrapping characteristic times of deep centers may play a role in the frequency dependence of the SNDC threshold bias as in the other phenomena related to traps. For example, Schulman [22] demonstrated that the presence of deep traps produces frequency dependence of the excess free and trapped carriers in a metal-semiconductor-metal structure and, consequently, leads to a frequency-dependent admittance. On the other hand, Ralph and Grischkowsky [23], in a $\mathrm{THz}$ time-domain spectroscopy (THz-TDS) system, showed a phenomenon consisting in an increase of the THz signal emitted by the photoconductive switch in the transition from the NDC to the breakdown regions, which appears in combination with an increase of the current. This is attributed to the trap-enhanced field at the anode region because of trap ionization. In our experiments, we find a similar threshold-like increase in the $\mathrm{THz}$ emitted signal but at the onset of the SNDC.

In this article, we study the effects of the NDC and the chopping frequency of the laser beam in the performance of photoconductive antennas fabricated on SI-GaAs. In a typical THz-TDS system, the induced photoconductive current at the receiver is in the order of picoampere, and so a low-frequency modulation is necessary for the lock-in detection of this small signal. We have chosen to chop the laser excitation, instead of the $\mathrm{THz}$ emitted signal, in order to modulate the generated carriers within the SI-GaAs and analyze the influence of the NDC-originated domains on the THz-TDS performance. We find that at the bias point where NDC emerges, which changes with the modulation frequency, a sharp increase in the current and the THz signal takes place. Additionally, NDC is found to be at the origin of a spurious signal in the detector that degrades the noise floor.
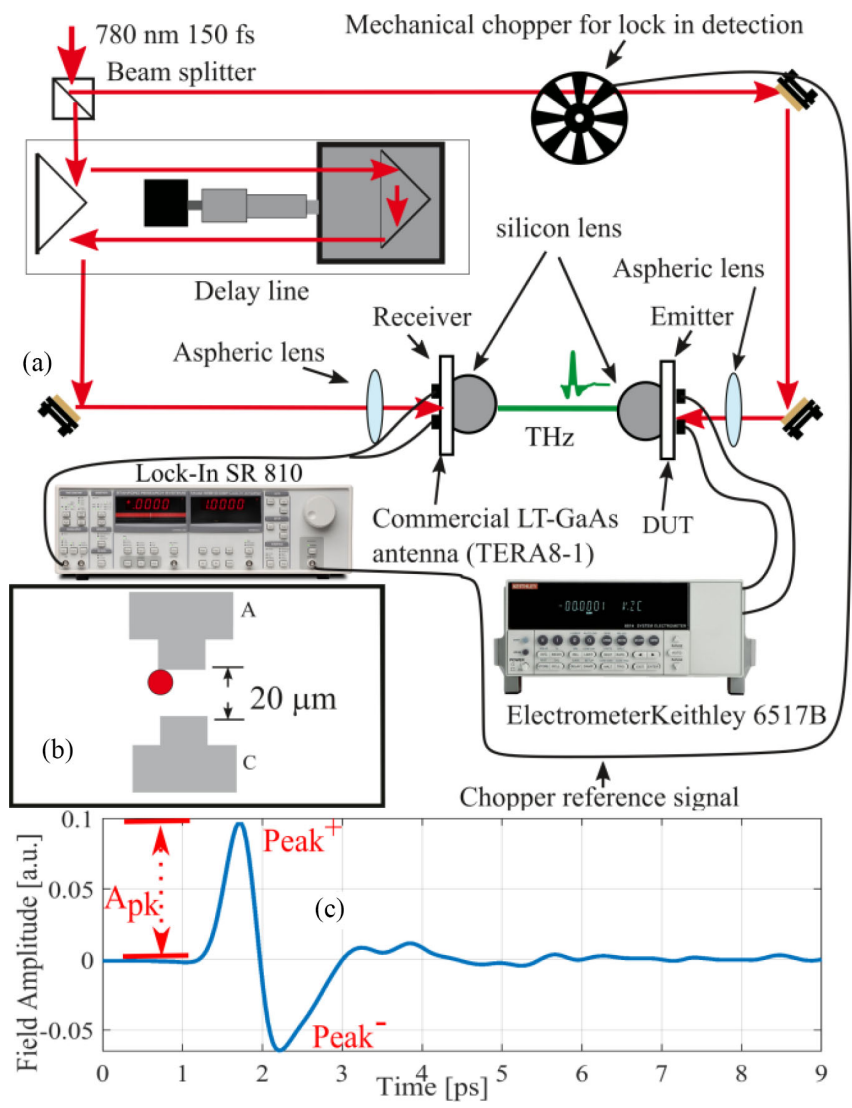

Fig. 1. (a) Experimental setup of the THz-TDS system; the Keithley 6517B electrometer is the voltage source for the emitter antenna (DUT). (b) Schematics of the DUT, with the red point representing the laser focused near the anode for optimal performance. (c) Detected $\mathrm{THz}$ pulse.

After Section I, the rest of this work is organized as follows. In Section II, we present the experimental setup and details about the fabrication of the sample. In Section III, the results are reported and discussed. The measurements of the current and THz signal as a function of the chopper frequency, as well as transient-current measurements, are provided, thus shedding light on the characteristic times of the phenomenon. Finally, Section IV presents the conclusions in this article.

\section{EXPERIMENTAL SETUP AND SAMPLE PREPARATION}

Fig. 1(a) shows the schematic of the THz-TDS system employed in the experiment. The infrared source is a MenloSystem 780 fiber laser. The wavelength is $780 \mathrm{~nm}$ with a $150 \mathrm{fs}$ pulsewidth and $100 \mathrm{MHz}$ of repetition rate. A beam splitter with 50:50 split ratio is used to divide the laser into the pump and the probe beams; a linear mechanical stage is the delay line necessary to make the correlation between the carriers generated at the receiver antenna by the probe beam and the $\mathrm{THz}$ electromagnetic pulse. The receiver is a TERA8-1 commercial LT-GaAs antenna fabricated by MenloSystems. An electrometer model Keithley 6517B is used as a voltage source and for current measurement. The mechanical chopper is the model SR540 from Stanford Research, which can modulate the pump beam up to 

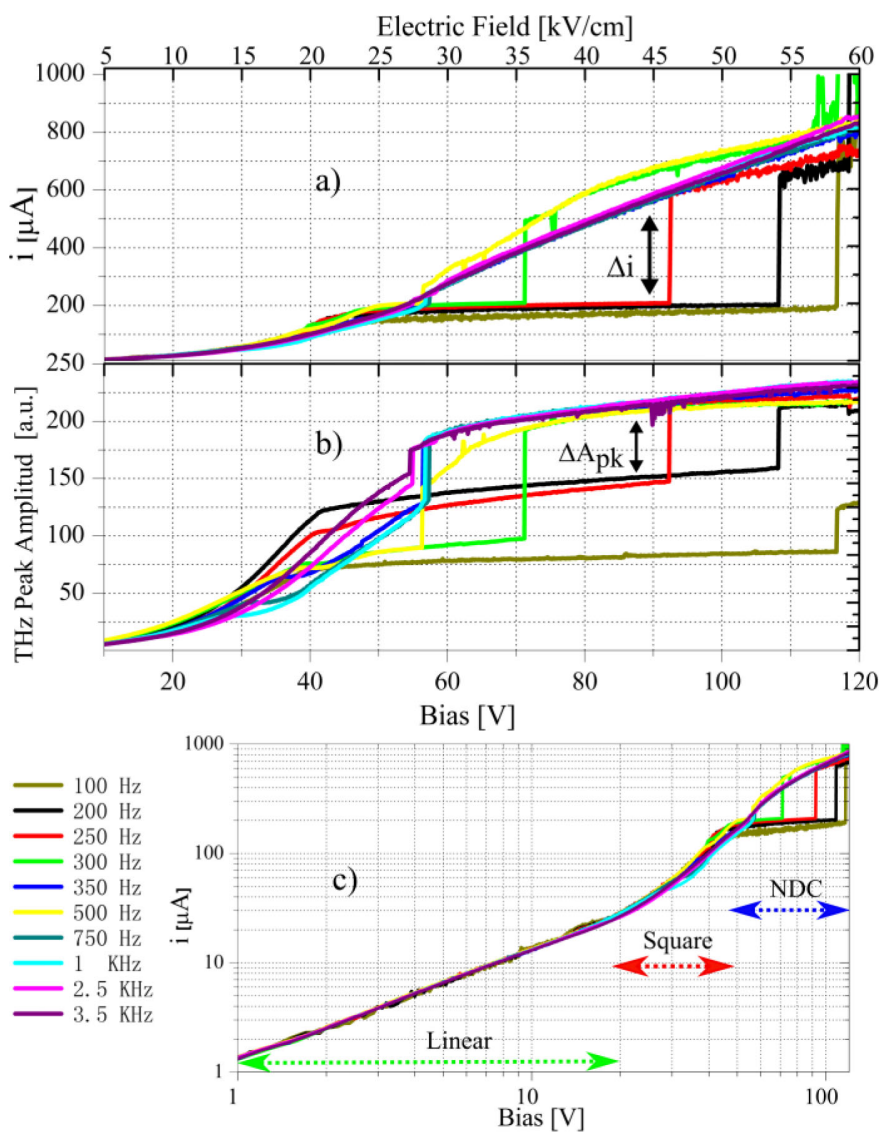

Fig. 2. (a) Current-voltage characteristic and (b) THz peak amplitude of the antenna with $20 \mu \mathrm{m}$ gap size measured under various chopping frequencies. The bottom $x$-axis corresponds to the bias voltages from 0 to $120 \mathrm{~V}$, with the bias resolution of $100 \mathrm{mV}$; the top $x$-axis corresponds to the equivalent electric field from 0 to $60 \mathrm{kV} / \mathrm{cm}$. (c) Current-voltage characteristics in logarithmic scale.

$4 \mathrm{kHz}$, and finally, the lock-in amplifier is the model SR810 from Stanford Research.

The emitter is a photoconductive antenna with a gap size of $20 \mu \mathrm{m}$ fabricated on a commercial (AXT, Inc.) SI-GaAs wafer with (100) orientation and dark resistivity of $1 \times 10^{7} \Omega \cdot \mathrm{cm}$. The native oxide on the surface of samples was removed with $1: 1 \mathrm{HCl}: \mathrm{H}_{2} \mathrm{O}$ for $1 \mathrm{~min}$; subsequently, the sample was passivated with hydrofluoric acid at $1 \%$ for $1 \mathrm{~min}$. The contact presents ohmic characteristics.

The laser is focused near the anode contact in order to obtain the strongest THz signal [23], as shown in Fig. 1(b). The estimated spot size of the focused beam is $5 \mu \mathrm{m}$. The device was tested by varying the applied voltage in steps of $100 \mathrm{mV}$ up to $120 \mathrm{~V}$. At each voltage value, the current feeding the photoconductive antenna and the positive peak amplitude [ $A_{\mathrm{pk}}$ in Fig. 1(c)] of the THz pulse were measured, and this phenomenon was repeated for increasing the frequencies of the chopper. All the tests were made under a constant laser average power of $10 \mathrm{~mW}$.

\section{RESULTS AND DISCUSSION}

Fig. 2 shows the current and the amplitude of the THz pulse $A_{\mathrm{pk}}$ for increasing chopping frequencies as a function of the

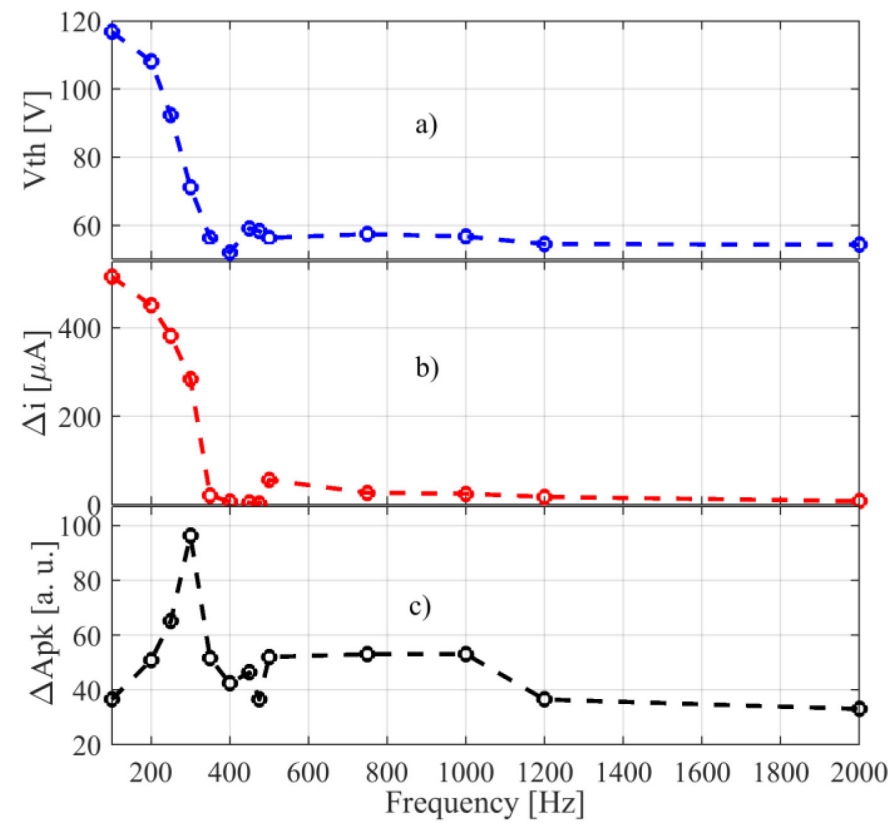

Fig. 3. (a) Threshold voltage $V_{\text {th }}$, (b) associated current increase $\Delta i$, and (c) THz peak amplitude increase $\Delta A_{\mathrm{pk}}$ as a function of the modulation frequency of the pump beam.

applied bias voltage. Fig. 2(c) shows the current in the log scale. For low bias, the current is linear up to around $25 \mathrm{~V}$ and then changes to a quadratic behavior. Beyond the quadratic region, the current and the $\mathrm{THz}$ peak amplitude saturate until a threshold bias $\left(V_{\mathrm{th}}\right)$ is reached, where the current and $\mathrm{THz}$ signal increase sharply. At low modulation frequencies, the step occurs at a higher value of the applied bias as compared with high modulation frequencies: $V_{\mathrm{th}}=115 \mathrm{~V}(57.5 \mathrm{kV} / \mathrm{cm})$ @ $100 \mathrm{~Hz}$, decreasing progressively until reaching a value of $55 \mathrm{~V}(27.5 \mathrm{kV} / \mathrm{cm}) @ 350 \mathrm{~Hz}$ and then not changing significantly with the further increase of the frequency. For frequencies below $350 \mathrm{~Hz}$, the current step at $V_{\text {th }}$ is quite large (for example, at $250 \mathrm{~Hz}$, the current changes from 197 to $595 \mu \mathrm{A}$ ) and the associated step in the THz signal is also remarkable; at $300 \mathrm{~Hz}$, the step in $A_{\mathrm{pk}}$ is $96 \mathrm{mV}$, in units of the lock-in amplifier. From Fig. 2(a) and (b), it is clear that the abrupt increase of the $\mathrm{THz}$ signal is perfectly correlated to the increase in the current, and there is a characteristic frequency around $350 \mathrm{~Hz}$ from which $V_{\text {th }}$ remains constant up to the maximum tested frequency (4 kHz). Fig. 3 shows the plots of $V_{\mathrm{th}}$, the current increase $\Delta i$ [see Fig. 2(a)], and the associated step change in the peak amplitude of the THz pulse $\Delta A_{\mathrm{pk}}$ as a function of the modulation frequency. The value of $V_{\mathrm{th}}$ decreases pronouncedly with the increase of frequency, from $117 \mathrm{~V}$ at $100 \mathrm{~Hz}$ to about $55 \mathrm{~V}$ at $350 \mathrm{~Hz}$, remaining nearly constant for higher frequencies. As observed, the maximum change of $A_{\mathrm{pk}}$ occurs at $300 \mathrm{~Hz}$. The step change in the current $\Delta i$ is high at low frequencies and then decreases with the increase of the modulation frequency, but above $300 \mathrm{~Hz}$, the step is much lower and nearly constant.

Subsequently, other experiments were conducted in order to achieve better understanding about the observed process. At this point, it is necessary to remark that the exact values of $V_{\text {th }}$ and 


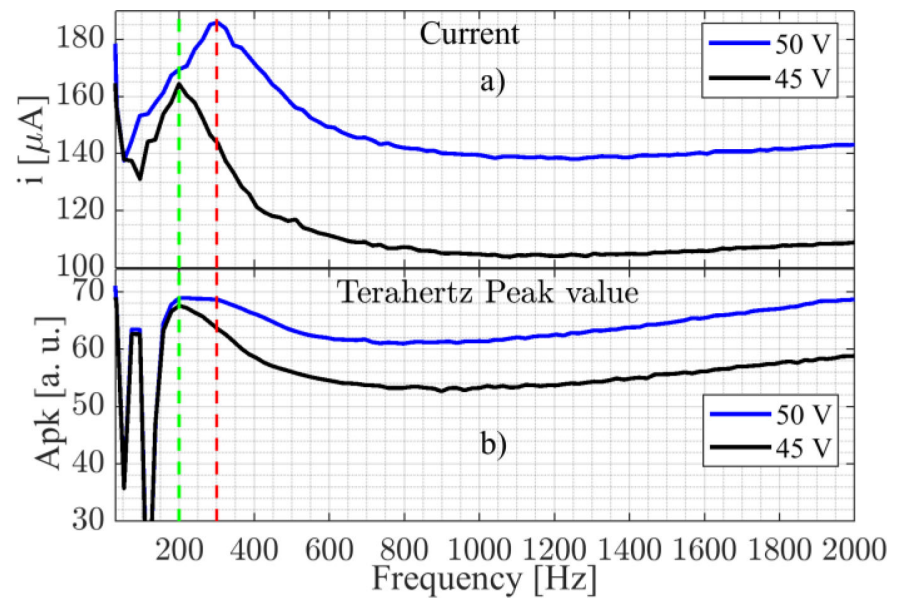

Fig. 4. (a) Photocurrent and (b) $\mathrm{THz}$ peak signal $\left(A_{\mathrm{pk}}\right)$ for a constant bias

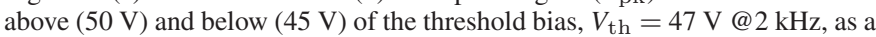
function of the chopping frequency.

$A_{\mathrm{pk}}$ show a strong dispersion from one experiment to another since they strongly depend on the specific placement of the laser on the antenna, the stress suffered by the specific sample and the influence of ambient conditions in optical mounts. In the following experiment, the device was biased at two voltages, lower and higher, respectively, than $V_{\text {th }}$ observed in the $I-V$ curve @ $2 \mathrm{kHz}$ (in this case, $47 \mathrm{~V}$, slightly lower than those previously shown). Both the current and $A_{\mathrm{pk}}$ were measured, while the chopping frequency was automatically varied in $10 \mathrm{~Hz}$ step from $30 \mathrm{~Hz}$ to $4 \mathrm{kHz}$. The data are presented in Fig. 4 as a function of the modulation frequency. For the bias of $45 \mathrm{~V}$, below $V_{\text {th }}$, the current (black curve) takes its highest value at $200 \mathrm{~Hz}$, the same frequency at which the $\mathrm{THz}$ amplitude is maximum. The deep notch observed in the THz signal at $120 \mathrm{~Hz}$ is due to the lock-in filter for the second harmonic of the power system. For the bias of $50 \mathrm{~V}$ (blue curve), above $V_{\mathrm{th}}$, both the current and the $\mathrm{THz}$ signal exhibit a maximum at $304 \mathrm{~Hz}$, a broad minimum around $900 \mathrm{~Hz}$, and then both increase monotonously. Remarkably, the current level is notably higher when the device is biased above $V_{\text {th }}$.

Fig. 5 shows the time shape of the THz pulse before and just after the step change in the current. For this run of the experiment, $V_{\mathrm{th}}=26.5 \mathrm{~V}$ and the modulation frequency $f_{\text {mod }}=2 \mathrm{kHz}$. The inset represents the spectrum of both pulses in a linear scale. As observed, the amplitude of the $\mathrm{THz}$ pulse and the corresponding peak at about $400 \mathrm{GHz}$ in the spectrum increase after the current step. In order to have a more precise comparison of the two conditions, the total energy of the time signals is determined by using the energy equation for discrete-time signals [24], obtaining an energy increase from 62 to 139 a.u., a 2.26 factor.

The sharp current increase observed in our experiments has close similarity with the $I-V$ characteristics resulting from conduction through the semi-insulating substrate in GaAs transistors with side gates [16], arising as a consequence of SNDC in a voltage-controlled experiment, as clearly explained by $\mathrm{Li}$ et al. [15]. We have, therefore, carried out a current-controlled experiment with the antennas to check if SNDC can be observed.

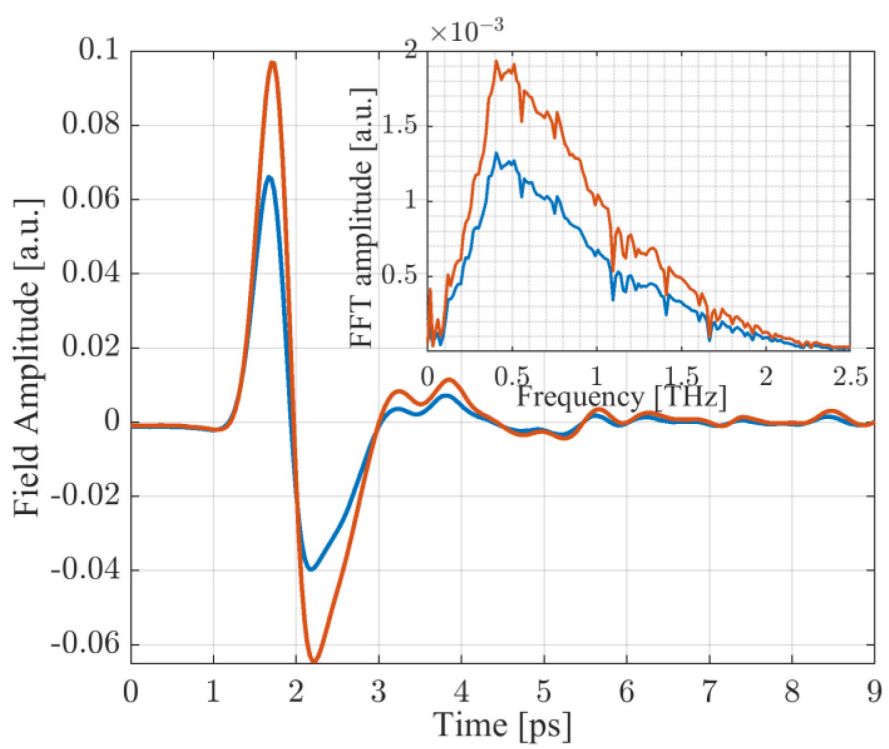

Fig. 5. THz pulses obtained for applied bias below (blue line) and above (red line) the sharp increase of the current for the case $V_{\mathrm{th}}=26.5 \mathrm{~V}$ and $f_{\text {mod }}=2 \mathrm{kHz}$. The inset shows their corresponding spectrum in linear scale.

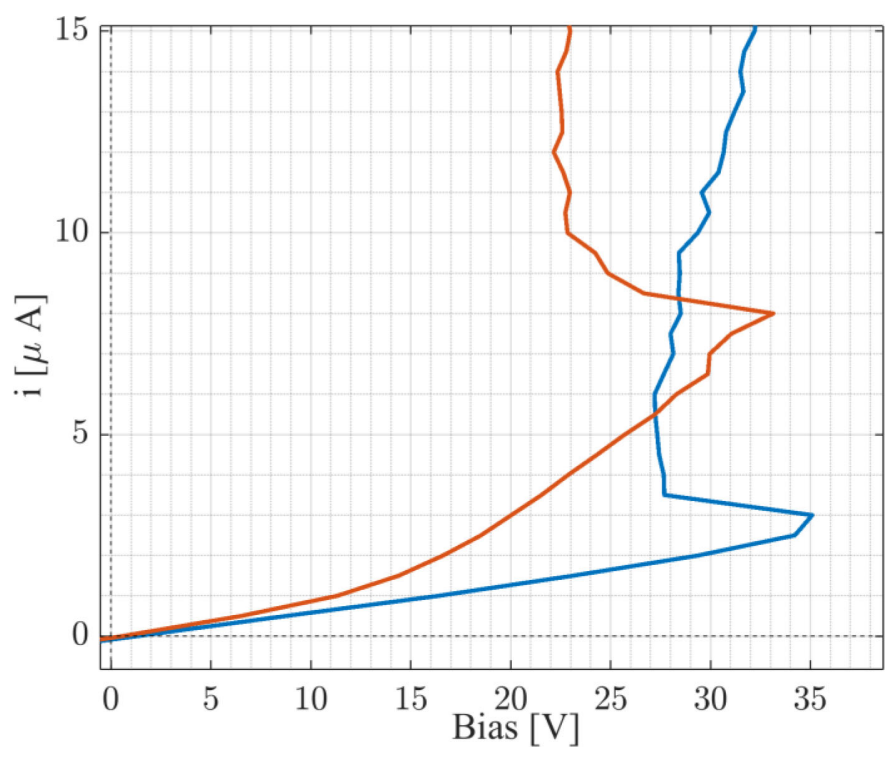

Fig. 6. SNDC observed in the photoconductive antenna with $20 \mu \mathrm{m}$ of gap size. The two curves correspond to consecutive experiments.

Using a Keithley SCS-4200 parameter analyzer, their $I-V$ characteristics were obtained in a current-controlled mode. Because of the high resistivity of the substrate, we used the light from a Leica microscope to slightly reduce the resistance of the device. Fig. 6 shows the $I-V$ curves obtained in two different current sweeps made on the same simple, which clearly exhibit SNDC. Both were measured consecutively, and their differences may be due to the variations of the ambient light during the experiment. This confirms the origin of the sharp step in the current and the $\mathrm{THz}$ emission observed in our samples.

An experiment using pulsed bias was also conducted in order to shed light on the characteristic time responsible for the chopping-frequency dependence observed in the measurements. 


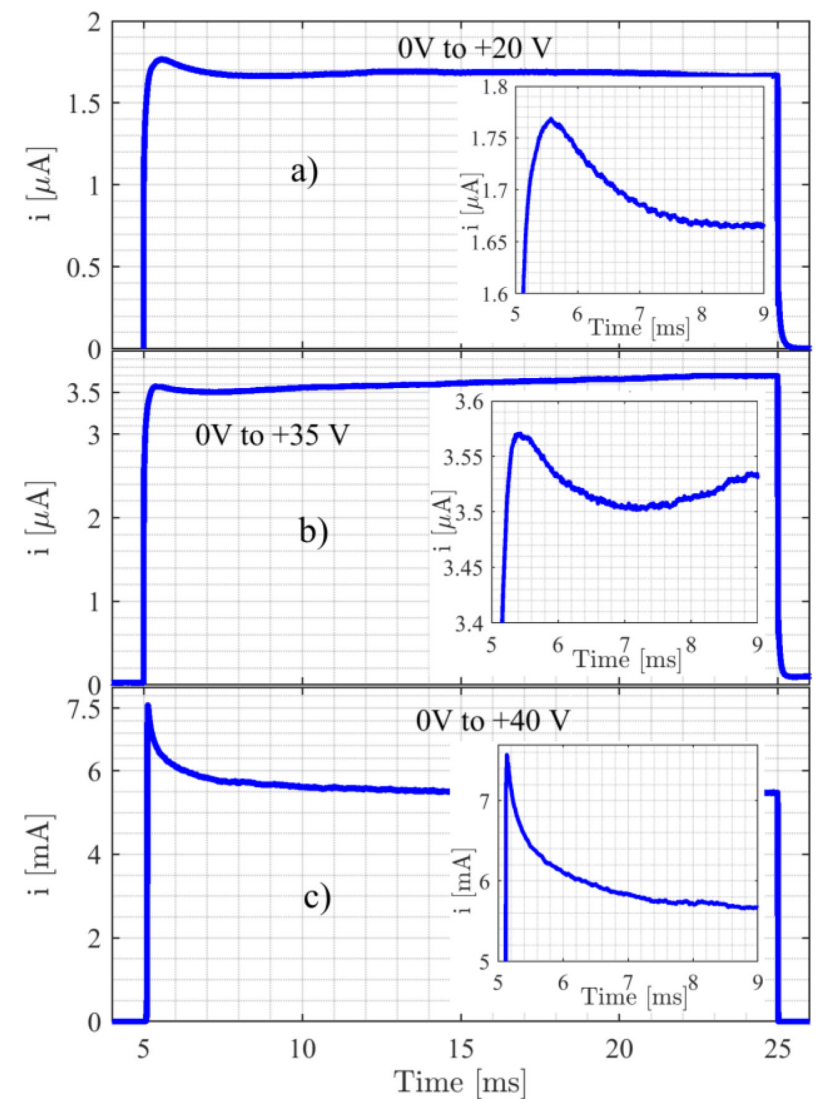

Fig. 7. Transient-current response of the photoconductive antenna with $20 \mu \mathrm{m}$ of gap size to pulse excitations. The amplitude of the pulses, applied at $5 \mathrm{~ms}$, is (a) $20 \mathrm{~V}$, (b) $35 \mathrm{~V}$, and (c) $40 \mathrm{~V}$; and the base voltage is $0 \mathrm{~V}$.

The device was biased with pulses of three different amplitudes, 20,35 , and $40 \mathrm{~V}$ (with a base voltage of $0 \mathrm{~V}$ ), and the transient currents were measured. The pulse bias is applied at time $t=$ $5 \mathrm{~ms}$. The transient-current curves are reported in Fig. 7. As observed, in the three cases, a transient of about $3 \mathrm{~ms}$ takes place before reaching the stationary value. Note that the inverse of this time is of the order of $300-350 \mathrm{~Hz}$, frequency around which $\Delta A_{\mathrm{pk}}$ takes its maximum value in Fig. 3, and also $A_{\mathrm{pk}}$ when measured beyond $V_{\text {th }}$ in Fig. 4. It is known that NDC can produce high-field domain regions, the origin of which is a drift or a generation-recombination nonlinearity [11]. The most accepted theory in the case of SI-GaAs is that NDC has its origin in a field-enhanced capture process. The transient-current measurements in response to step voltages, as presented in Fig. 7, provide information about the deep-level trapping mechanisms. The measured characteristic times in the sample, of the order of 2.5-3 ms, are within the range of the observed frequency dependence of the abrupt transition in the $\mathrm{THz}$ signal and the current of the antenna, thus confirming the trap-related origin of the phenomenon.

In works dealing with chaos in photoconductors, the emission of broadband noise at the NDC region has been observed [21], [25]. Our experiments indicate that at the bias corresponding to the onset of NDC, the emitter produces an additional signal that becomes an important source of noise at the receiver. As a consequence, the pulse scanned at the receiver side presents
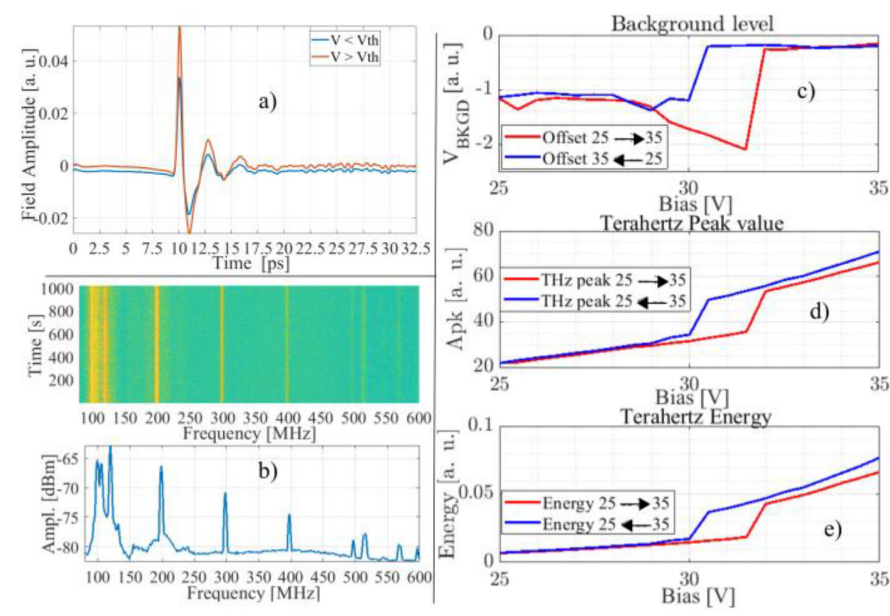

Fig. 8. (a) THz pulse with an increase of the background level (spurious signal) at the threshold bias. (b) Spectrum of the signal entering to the lock-in amplifier. (c) Background level, (d) THz peak amplitude, and (e) energy of each individual pulse as a function of the bias of the antenna between 25 and $35 \mathrm{~V}$ swept in both directions.

this spurious signal, as observed in Fig. 8(a). This slow-varying spurious signal is superimposed to the pulse so that the effect on the $\mathrm{THz}$ pulse is perceived as a shift in the background level. This additional phenomenon has its origin in the laser excitation; thus, it is unavoidably modulated at the same chopping frequency and, hence, detected with the lock-in amplifier. The spectrum of the induced signal at the receiver obtained with a Keysight N9344C spectrum analyzer, as shown in Fig. 8(b), shows that the laser repetition rate $(100 \mathrm{MHz})$ and its harmonics are present, but there are other (unknown) slow signals that have frequency components at 106 and $120 \mathrm{MHz}$. The chopping frequency in this test is $2 \mathrm{kHz}$. This background signal could be originated by the presence of slowly propagating high-field domains in the antenna originated by the SNDC.

An additional experiment was conducted; instead of just measuring the peak amplitude, the entire pulse is recorded in a temporal window of 66 ps. Fig. 8(c)-(e) shows the results corresponding to the background level of each pulse, the peak amplitude $A_{\mathrm{pk}}$, and the total energy of each individual pulse. The bias voltage is swept in both directions, the red lines correspond to an increase of the bias from 25 to $35 \mathrm{~V}$, where the threshold voltage $V_{\text {th }}$ is found to be $31.5 \mathrm{~V}$, and the blue lines to a decrease from 35 to $25 \mathrm{~V}$, with a $V_{\text {th }}$ of $30 \mathrm{~V}$ in this case. The chopper frequency in this experiment is $2 \mathrm{kHz}$. Similarly to the results shown in the work of Li et al. [15], the device with SNDC presents hysteresis depending on the direction in which the voltage is swept (in our case, all the three parameters considered). Concurrently, with the $\mathrm{THz}$ signal increase at $V_{\mathrm{th}}$, the background level also increases, which introduces uncertainly in the measurements made with the THz-TDS system.

\section{CONCLUSION}

Deep traps confer interesting electrical properties to SI-GaAs. The main one is the reduction of the carrier lifetime, the key to the generation of pulsed $\mathrm{THz}$ radiation. But their presence 
also produces other complex phenomena, such as SNDC, that can influence the performance of the devices, as shown by our results. In THz-TDS systems, using photoconductive antennas, normally it is necessary the chopping of the excitation laser beam or the output $\mathrm{THz}$ signal. If the laser is modulated, it has a direct influence on the system because the photoconductive antennas present SNDC at a given threshold bias. At this point, the static $I-V$ curve presents a sharp current increase in a voltagecontrolled experiment, which is reflected in the $\mathrm{THz}$ pulse as an increase of the peak amplitude. The calculated energy after the transition is double that of the pulse before this phenomenon takes place. Remarkably, the threshold bias changes with the modulation frequency of the laser beam as long as such frequency is below $350 \mathrm{~Hz}$.

The trapping and detrapping characteristic time of deep centers is the responsibility of the frequency dependence of the threshold bias for SNDC and the related effects. The transient measurements using pulsed-bias excitations provide characteristic times coincident with the maximum frequency at which the variations of the threshold bias are observed. Our experiments also demonstrate that NDC in the emitter is an important source of noise at the receiver. Beyond the threshold bias, a slow-varying spurious signal (with a much longer characteristic time than the picosecond $\mathrm{THz}$ pulse) is present in the receiver, thus modifying the background level of the signal, which can be an important source of uncertainty in applications where quantitative measurements are performed with the THz-TDS.

We must finally remark that NDC in the photoconductive antenna, and all the associated effects, could be avoided by mechanically chopping the $\mathrm{THz}$ signal instead of the laser beam since the laser repetition rate is too high to lead to the formation of high-field domains originated by the field-enhanced EL2 capture of electrons.

\section{ACKNOWLEDGMENT}

The authors would like to thank I. Juárez-Ramírez for the assistance in the devices wire bonding and $\mathrm{O}$. Aponte-Bravo for technical assistance in the cleanroom.

\section{REFERENCES}

[1] N. M. Burford and M. O. El-Shenawee, "Review of terahertz photoconductive antenna technology," Opt. Eng., vol. 56, no. 1, Jan. 2017, Art. no. 010901.

[2] A. Singh et al., "Carbon irradiated semi insulating GaAs for photoconductive terahertz pulse detection," Opt. Express, vol. 23, no. 5, pp. 6656-6661, Mar. 2015.

[3] C. Sun and A. Zhang, "Ion-beam modified terahertz GaAs photoconductive antenna," in Advances in Photodetectors-Research and Applications. London, U.K.: IntechOpen, 2019.

[4] J. Madéo et al., "Ultrafast properties of femtosecond-laser-ablated GaAs and its application to terahertz optoelectronics," Opt. Lett., vol. 40, no. 14, pp. 3388-3391, Jul. 2015.

[5] G. Kühnel, W. Siegel, and H. A. Schneider, "High-field current-voltage characteristics of semi-insulating GaAs," Physica Status Solidi, vol. 100, no. 1, pp. 283-287, Mar. 1987.

[6] J. J. Mareš et al., "High field transport in semi-insulating GaAs: A promising material for solid-state detectors," J. Appl. Phys., vol. 82, no. 7, pp. 3358-3362, Oct. 1997.

[7] B. Willing and J. C. Maan, "Non-linear transport and structure formation in semi-insulating GaAs," J. Phys., Condens. Matter, vol. 8, no. 40, pp. 7493-7508, Sep. 1996.
[8] H. A. Albuquerque et al., "Impact ionization and field-enhanced trapping: Fitting current density curves for semi-insulating GaAs," J. Appl. Phys., vol. 93, no. 3, pp. 1647-1650, Feb. 2003.

[9] C. Paracchini and V. Dallacasa, "Impact ionization current in semiinsulating GaAs," Solid State Commun., vol. 69, no. 1, pp. 49-52, Jan. 1989.

[10] C. Groves, R. Ghin, J. P. R. David, and G. J. Rees, "Temperature dependence of impact ionization in GaAs," IEEE Trans. Electron Devices, vol. 50, no. 10, pp. 2027-2031, Oct. 2003.

[11] F. Piazza, P. C. M. Christianen, and J. C. Maan, "Propagating high-electricfield domains in semi-insulating GaAs: mExperiment and theory," Phys. Rev. B, vol. 55, no. 23, pp. 15591-15600, Jun. 1997.

[12] A. Neumann, "Slow domains in semi-insulating GaAs," J. Appl. Phys., vol. 90 , no. 1, pp. 1-26, 2001.

[13] F. Piazza, P. C. M. Christianen, and J. C. Maan, "Electric field dependent EL2 capture coefficient in semi-insulating GaAs obtained from propagating high field domains," Appl. Phys. Lett., vol. 69, no. 13, pp. 1909-1911, Sep. 1996.

[14] E. Schöll, "Instabilities in semiconductors: Domains, filaments, chaos," in Festkörperprobleme, vol. 26, P. Grosse, Ed. Berlin, Germany: Springer, 1986, pp. 309-333.

[15] Z.-M. Li, S. P. McAlister, W. G. McMullan, C. M. Hurd, and D. J. Day, "Impact ionization of deep traps in semi-insulating GaAs substrates," $J$. Appl. Phys., vol. 67, no. 12, pp. 7368-7372, Jun. 1990.

[16] Y. Ohno and N. Goto, "Mechanism of electrostatic potential conduction in semi-insulating substrates," J. Appl. Phys., vol. 66, no. 3, pp. 1217-1221, Aug. 1989.

[17] H. K. Sacks and A. G. Milnes, "Low frequency oscillations in semiinsulating gallium arsenide †," Int. J. Electron., vol. 28, no. 6, pp. 565-583, Jun. 1970.

[18] U. Rau et al., "Reconstruction of traveling waves in semi-insulating GaAs," Phys. Lett. A, vol. 152, no. 7, pp. 356-360, Jan. 1991.

[19] H. Rajbenbach, J. M. Verdiell, and J. P. Huignard, "Visualization of electrical domains in semi-insulating $\mathrm{GaAs}: \mathrm{Cr}$ and potential use for variable grating mode operation," Appl. Phys. Lett., vol. 53, no. 7, pp. 541-543, Aug. 1988.

[20] H. C. Ellin, A. Grunnet-Jepsen, and L. Solymar, "Generation and inhibition of domain movement in semi-insulating GaAs:Cr," Appl. Phys. Lett., vol. 65, no. 3, pp. 353-355, Jul. 1994.

[21] S. W. Teitsworth and R. M. Westervelt, "Chaos and broadband noise in extrinsic photoconductors," Phys. Rev. Lett., vol. 53, no. 27, pp. 2587-2590, Dec. 1984.

[22] D. D. Shulman, "Low-frequency transport in semi-insulating GaAs," $J$. Appl. Phys., vol. 72, no. 6, pp. 2288-2293, Sep. 1992.

[23] S. E. Ralph and D. Grischkowsky, "Trap-enhanced electric fields in semiinsulators: The role of electrical and optical carrier injection," Appl. Phys. Lett., vol. 59, no. 16, pp. 1972-1974, Oct. 1991.

[24] L. Chaparro, Signals and Systems Using MATLAB. Burlington, MA, USA: Academic, 2010.

[25] S. W. Teitsworth and R. M. Westervelt, "Subharmonic and chaotic response of periodically driven extrinsic Ge photoconductors," Phys. Rev. Lett., vol. 56, no. 5, pp. 516-519, Feb. 1986.

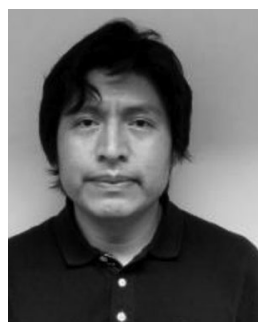

Gaudencio Paz-Martínez received the Ph.D. degree in electrical engineering from the Universidad $\mathrm{Na}$ cional Autónoma de México, Mexico City, Mexico, in 2015.

$\mathrm{He}$ is currently a Postdoctoral Researcher with the Department of Applied Physics, University of Salamanca, Salamanca, Spain. His research interests include terahertz electronics and photonics.

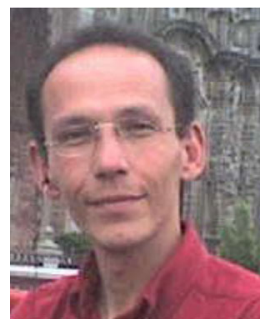

Carlos Gerardo Treviño-Palacios received the B.Sc. degree in Physics from the Universidad Nacional Autónoma de México, Mexico City, Mexico, and the Ph.D. degree in Optical Science and Engineering from the Center for Research and Education in Optics and Lasers, University of Central Florida, Orlando, FL, USA, in 1998.

$\mathrm{He}$ is currently the Head of a laboratory dedicated to nonlinear optics, laser science, and terahertz photonics, Instituto Nacional de Astrofísica, Óptica y Electrónica, Puebla, Mexico. 


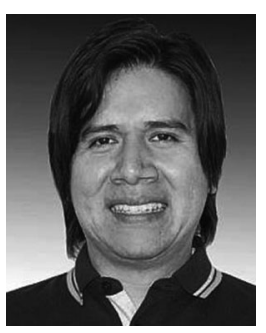

Joel Molina-Reyes (Senior Member, IEEE) received the B.S.E.E. degree (magna cum laude) from Universidad Veracruzana, Veracruz, Mexico, the M.Sc. degree in microelectronics from the National Institute for Astrophysics, Optics, and Electronics (INAOE), Puebla, Mexico, and the Ph.D. degree in advanced applied electronics from the Tokyo Institute of Technology, Tokyo, Japan.

He is currently a Full Professor with INAOE. His research interests include the physics and technology of complementary metal-oxide-semiconductorcompatible electron devices based on the ultrathin metal and high dielectric constant insulators for application in advanced logic, memory, sensing, microelectromechanical systems, novel nanostructured materials, and photoactive technologies. He has authored or coauthored more than 100 peer-reviewed research publications.

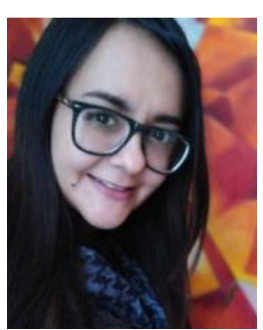

Alejandra Romero-Morán received the bachelor's degree in chemistry from the Universidad de las Americas Puebla, Cholula, Mexico, in 2010, the M.Sc. degree in chemical sciences from the Benemérita Universidad Autónoma de Puebla, Puebla, Mexico, in 2015, and the Ph.D. degree in electronics from the National Institute for Astrophysics, Optics, and Electronics, Puebla, Mexico, in 2020.

Her research interests include material science, photocatalysis, and environmental science.

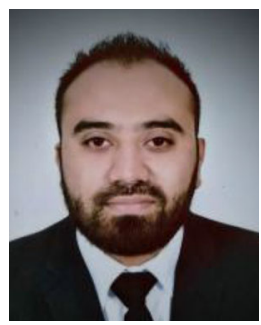

Eric Cervantes-García received the B.Eng. in electronic and communication systems engineering from the Universidad Autónoma de la Ciudad de México, Mexico City, Mexico, in 2017, and the M.Sc. degree in Space Science and Technology from the Instituto Nacional de Astrofísica, Óptica y Electrónica, Puebla, Mexico, in 2017, where he is currently working toward the Ph.D. degree in Optics with the Department of Optics.

His current research interests include terahertz photonics and high-frequency devices.

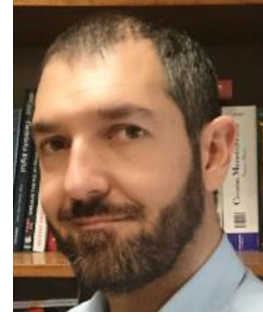

Javier Mateos (Member, IEEE) was born in Salamanca, Spain, in 1970. He received the B.S. and Ph.D. degrees in physics from the University of Salamanca, Salamanca, Spain, in 1993 and 1997, respectively.

He is currently a Full Professor of electronics with the Department of Applied Physics, University of Salamanca. He has a long experience in the Monte Carlo modeling of the high-frequency and low-noise performance of III-V high-electron mobility transistors, and his main present research interest is in the development of novel device concepts for terahertz generation and detection. He is the Author or Co-Author of more than 130 refereed scientific journal papers and 200 conference contributions.

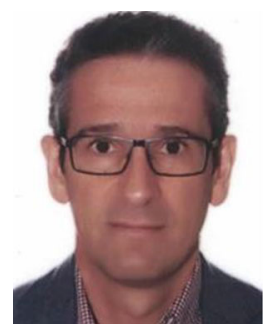

Tomás González (Senior Member, IEEE) received the Graduate and Ph.D. degrees in physics from the University of Salamanca, Salamanca, Spain, in 1990 and 1994, respectively.

Since 1991, he has been with the Department of Applied Physics, University of Salamanca, where he is currently a Full Professor of electronics and leads the Research Group on high-frequency nanoelectronic devices. In the fall of 1992 , he was a Visiting Researcher with the Department of Physics, University of Modena and Reggio Emilia, Modena and Reggio Emilia, Italy. His main research activities include the fields of device modeling, high-frequency III-V transistors, and terahertz electronics. $\mathrm{He}$ is Author or Co-Author of more than 170 refereed scientific journal papers and 250 conference contributions.

Dr. González has been an Associate Editor for the Journal of Computational Electronics since 2012, Guest Editor of special issues in several international journals, and serves on the committees of various international conferences (ICNF and EDISON). 